\title{
Correction to: Final Conclusion and Proposal
}

\section{Correction to:}

\section{Chapter 5 in: K. Abhijeet, National Space Legislation for India, https://doi.org/10.1007/978-981-15-2675-6_5}

In the original version of the book, Section 5.2 of Chapter 5 has been changed from "Draft National Space Legislation 201_" to "Draft National Space Legislation 202_". The chapter and the book have been updated with the change. 